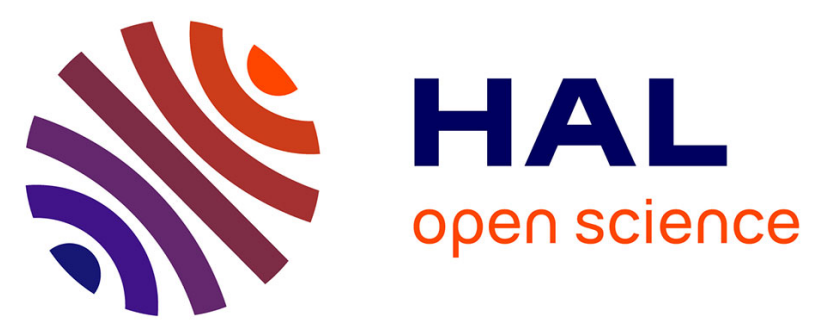

\title{
Accelerated senescence of cord blood endothelial progenitor cells in premature neonates is driven by SIRT1 decreased expression
}

\author{
Paula Frizera Vassallo, Stephanie Simoncini, Isabelle Ligi, Anne-Line \\ Château, Richard Bachelier, Stéphane Robert, Julia Morere, Samantha \\ Fernandez, Benjamin Guillet, Maxime Marcelli, et al.
}

\section{To cite this version:}

Paula Frizera Vassallo, Stephanie Simoncini, Isabelle Ligi, Anne-Line Château, Richard Bachelier, et al.. Accelerated senescence of cord blood endothelial progenitor cells in premature neonates is driven by SIRT1 decreased expression. Blood, 2014, 123 (13), pp.2116 - 2126. 10.1182/blood-2013-02-484956 . hal-01666068

\section{HAL Id: hal-01666068 \\ https://hal-amu.archives-ouvertes.fr/hal-01666068}

Submitted on 18 Dec 2017

HAL is a multi-disciplinary open access archive for the deposit and dissemination of scientific research documents, whether they are published or not. The documents may come from teaching and research institutions in France or abroad, or from public or private research centers.
L'archive ouverte pluridisciplinaire HAL, est destinée au dépôt et à la diffusion de documents scientifiques de niveau recherche, publiés ou non, émanant des établissements d'enseignement et de recherche français ou étrangers, des laboratoires publics ou privés. 


\title{
VASCULAR BIOLOGY
}

\section{Accelerated senescence of cord blood endothelial progenitor cells in premature neonates is driven by SIRT1 decreased expression}

\author{
Paula Frizera Vassallo, ${ }^{1}$ Stéphanie Simoncini, ${ }^{1}$ Isabelle Ligi, ${ }^{1,2}$ Anne-Line Chateau, ${ }^{1}$ Richard Bachelier, ${ }^{1}$ Stéphane Robert, ${ }^{1}$ \\ Julia Morere, ${ }^{3}$ Samantha Fernandez, ${ }^{4}$ Benjamin Guillet, ${ }^{1,4}$ Maxime Marcelli, ${ }^{5}$ Edwige Tellier, ${ }^{1}$ Alain Pascal, ${ }^{6}$ \\ Umberto Simeoni, ${ }^{1,2}$ Francine Anfosso, ${ }^{1}$ Frédérique Magdinier, ${ }^{3}$ Françoise Dignat-George, ${ }^{1,7}$ and Florence Sabatier ${ }^{1,8}$ \\ ${ }^{1}$ Aix-Marseille Université, INSERM, Vascular Research Center of Marseille, Unité Mixte de Recherche en Santé 1076, Marseille, France; ${ }^{2}$ Département de \\ Néonatologie, Assistance Publique Hôpitaux de Marseille, Centre Hospitalier Universitaire de la Conception, Marseille, France; ${ }^{3}$ Aix-Marseille Université, \\ INSERM, Génétique Médicale et Génomique Fonctionnelle, Unité Mixte de Recherche en Santé 910, Marseille, France; ${ }^{4}$ Aix-Marseille Université, Centre \\ Européen de Recherche en Imagerie Médicale, Marseille, France; ${ }^{5}$ Département d'Obstétrique, Assistance Publique Hôpitaux de Marseille, Centre Hospitalier \\ Universitaire de la Conception, Marseille, France; ${ }^{6}$ Service d'Obstétrique, Hôpital Saint Joseph, Marseille, France; ${ }^{7}$ Service d'Hématologie, Assistance \\ Publique Hôpitaux de Marseille, Centre Hospitalier Universitaire de la Conception, Marseille, France; and ${ }^{8}$ Assistance Publique Hôpitaux de Marseille, Centre \\ Hospitalier Universitaire de la Conception, Laboratoire de Culture et Thérapie Cellulaire, INSERM, Centres d'Investigation Clinique-BT510, Marseille, France
}

\section{Key Points}

- We demonstrate that PT promotes ECFCs dysfunction by inducing stress-induced premature senescence.

- Our data reveal that SIRT1 deficiency drives PT-ECFC senescence, and acts as a critical determinant of the PT-ECFC angiogenic defect.

Epidemiological and experimental studies indicate that early vascular dysfunction occurs in low-birth-weight subjects, especially preterm (PT) infants. We recently reported impaired angiogenic activity of endothelial colony-forming cells (ECFCs) in this condition. We hypothesized that ECFC dysfunction in PT might result from premature senescence and investigated the underlying mechanisms. Compared with ECFCs from term neonates $(n=18)$, ECFCs isolated from PT $(n=29)$ display an accelerated senescence sustained by growth arrest and increased senescence-associated $\beta$-galactosidase activity. Increased p16 ${ }^{\text {INK4a }}$ expression, in the absence of telomere shortening, indicates that premature PT-ECFC aging results from stress-induced senescence. SIRT1 level, a nicotinamide adenine dinucleotidedependent deacetylase with anti-aging activities, is dramatically decreased in PT-ECFCs and correlated with gestational age. SIRT1 deficiency is subsequent to epigenetic silencing of its promoter. Transient SIRT1 overexpression or chemical induction by resveratrol treatment reverses senescence phenotype, and rescues in vitro PT-ECFC angiogenic defect in a SIRT1-dependent manner. SIRT1 overexpression also restores PT-ECFC capacity for neovessel formation in vivo. We thus demonstrate that decreased expression of SIRT1 drives accelerated senescence of PT-ECFCs, and acts as a critical determinant of the PT-ECFC angiogenic defect. These findings lay new grounds for understanding the increased cardiovascular risk in individuals born prematurely and open perspectives for therapeutic strategy.

\section{Introduction}

Accumulating evidence indicates that the perinatal environment contributes to the risk for noncommunicable diseases later in life. In this instance, low birth weight, in particular preterm (PT) birth, is considered as a major independent risk factor for cardiovascular-related diseases, ${ }^{1}$ increased risk of hypertension, altered glucose tolerance, or renal dysfunction at adulthood. This fetal programming might influence cardiovascular risk by adversely acting at different levels on the vascular bed including reduction in arterial stiffness, microvascular density due to incomplete vasculogenesis, and impairment of endothelial function. ${ }^{2,3}$ However, the underlying mechanisms linking alterations of vascular function related to PT birth to increased risk of late cardiovascular disease remain unclear. ${ }^{2}$

Under physiological conditions, endothelial repair is achieved through the contribution of endothelial progenitor cells, derived from the bone marrow. ${ }^{4,5}$ Among them, a specific subtype called endothelial colony-forming cells (ECFCs) has been recently characterized both in cord and peripheral blood. These cells emerge as adherent colonies after culture of mononuclear cells (MNC) and develop as a typical endothelial monolayer. ECFCs harbor clonogenic potential, endothelial phenotype, have the capacity to incorporate into neovessels, and participate in the maintenance of vascular homeostasis in vivo. Impairment in the number and function of ECFCs is observed in several diseases associated with cardiovascular risk such as diabetes, renal failure, atherosclerosis, or preeclampsia. ${ }^{6-8}$ Therefore, adult blood ECFCs emerge as key effectors and potential biological marker for a variety of cardiovascular diseases. Nevertheless, few studies have focused on cord blood EPCs in pathological conditions. Quantitative and functional abnormalities of cord blood ECFCs have been evidenced not only in preeclampsia or type 2 diabetes mellitus, ${ }^{9-11}$ but also in vascular pathologies such as bronchopulmonary dysplasia. ${ }^{12}$ 
Cellular senescence plays a key role in the development of cardiovascular diseases associated with aging. Indeed, senescence is not only associated with endothelial cell dysfunction, ${ }^{13,14}$ but also impaired angiogenesis. ${ }^{15,16}$ Senescent endothelial cells have been detected in human atherosclerotic tissues and exhibit various functional abnormalities. ${ }^{14}$ Moreover, diabetes in vivo and glycated collagen exposure in vitro elicit premature senescence of the vascular endothelium and angiogenesis defect. ${ }^{15}$ Interestingly, in ECFCs from low-birth-weight PT infants compared with infants born at term, with a normal weight, ${ }^{17}$ we previously demonstrated a striking reduced angiogenic potential with a switch toward angiostatic factors suggesting that ECFC cellular fitness is impaired in infants born prematurely.

Herein, we hypothesized that the senescence process could limit the ability of PT ECFCs to sustain endothelial repair. We demonstrate for the first time that PT birth promotes ECFC dysfunction by inducing stress-induced premature senescence. This senescence is driven by decreased expression of SIRT1, a nicotinamide adenine dinucleotide-dependent deacetylase possessing anti-aging activities. Interestingly, upregulation of SIRT1, after transient overexpression or resveratrol (RSV) treatment, prevents PT-induced ECFCs senescence and reverses angiogenic defects in ECFCs from premature infants, both in vitro and in vivo.

\section{Patients, materials, and methods}

An expanded Materials and methods section is available in the supplemental Methods (available on the Blood Web site). This study was conducted in accordance with the Declaration of Helsinki.

\section{Culture of ECFCs}

ECFCs were isolated from the MNC fraction obtained from the cord blood of term (CT) and PT neonates, cultured, and characterized as previously described. ${ }^{17}$

\section{SA- $\beta$-gal staining}

Senescence-associated- $\beta$-galactosidase (SA- $\beta$-gal) activity was performed using a senescence detection kit (BioVision Research Products) according to the manufacturer's instructions. The percentage of SA- $\beta$-gal-positive cells was counted in 10 randomly selected microscopic fields (magnification $\times 20$; 400-600 cells).

\section{Proliferation assay and cell-cycle analysis}

Proliferative capacity was analyzed using a bromodeoxyuridine (BrdU) incorporation assay (Roche Molecular Biochemicals) according to the manufacturer's instructions. For cell-cycle analysis, ECFCs were submitted to a starving period of 24 hours with EBM2 containing $0.5 \%$ fetal calf serum, then incubated for 24 hours with EGM2-MV medium, harvested, fixed with $70 \%$ cold ethanol, and stained with propidium iodide. At least 10000 events were acquired per sample with a Gallios flow cytometer system and analyzed using Kaluza Analysis software (Beckman Coulter).

\section{Drug treatment}

ECFCs were incubated with $1 \mu \mathrm{M}$ RSV (Calbiochem) resuspended in dimethylsulfoxide (DMSO). Control cells were mock-treated with DMSO. For nicotinamide (NAM), medium containing 1mM NAM was added and cells were incubated for an additional 24 hours before harvesting for SA- $\beta$-gal staining or 3-dimensional (3D) spheroid assay, or 48 hours for BrdU assays.

\section{Plasmids transfection}

Cells were transfected with the pCMV-Sport6 expression vector (Empty) or the pCMV-Sport6-SIRT1 plasmid (NIH_MGC_91 clone) using the jetPRIME in vitro DNA transfection protocol (Polyplustransfection SA).

\section{Antibodies}

Antibodies against $\mathrm{p} 16^{I N K 4 a}$, SIRT1, p21 ${ }^{W A F}, \mathrm{p} 53$, acetyl-p53 (Lys382), phospho-retinoblastoma (P-Rb) (Ser795), Rb (4H1), and $\beta$-Tubulin (9F3) were purchased from Cell Signaling Technology and used at the recommended dilution for immunoblotting ( $1 / 2000$ for $\mathrm{Rb}$ and $1 / 1000$ for the others). Anti- $\beta$ actin was purchased from Neomarkers and used at a 1/2000 dilution.

\section{RNA isolation and quantitative RT-PCR}

Total RNA was extracted using the mirVana miRNA Isolation Kit (Ambion). Quantitative reverse transcription-polymerase chain reaction (RT-PCR) was performed using the Brillant QPCR Master Mix (Stratagene) using predesigned primers for p16 ${ }^{I N K 4 a}$ (HS00233365_m1), SIRT1 (HS01009003_m1), p21 ${ }^{\text {WAF }}$ (HS00355782_m1), p53(HS01034249_m1), and RPL13 (HS00204173_m1) (Applied Biosystems). Relative fold-change was determined using the $2-\Delta \Delta^{\mathrm{CT}}$ method with ECFCs as the baseline and normalization to RPL13A expression.

\section{Angiogenesis testing}

Tube formation. ECFCs were cultured at 20000 cells per well in 96-well plates coated with $50 \mu \mathrm{L}$ of growth factor-reduced Matrigel (BD Biosciences). Capillary-like structures were counted using ImageJ software (NCBI). Each experiment was done in triplicate.

Spheroid-based sprouting assay. ECFCs were seeded as previously described and incubated or not with $1 \mu \mathrm{M} \mathrm{RSV} .{ }^{18}$ After 30 minutes, $100 \mu \mathrm{L}$ of EGM-2 MV with either DMSO, $1 \mu$ M RSV, or with RSV and $1 \mathrm{mMNAM}$ was added to the surface of each well. After 24 hours, 20 spheroids were analyzed for each independent sample using a Nikon microscope at a $\times 20$ magnification. Cumulative sprout length (per spheroid), number of sprouts, and ramifications were quantified using the Image $\mathbf{J}$ software.

In vivo Matrigel plug assay. Growth factor-reduced Matrigel was mixed either with DMSO or 1mM RSV and filled with a mixture (75:25) of ECFCs and a commercially available human umbilical artery smooth muscle cells (Promocell).The plugs were subcutaneously injected in the groin lateral areas of nu/nu mice. After 1 week, mice received $50 \mu \mathrm{L}$ of $1 \mathrm{mg} / \mathrm{mL}$ Alexa Fluor 488-dextran (Molecular Probes) in a physiological $\mathrm{NaCl}$ solution IV 20 minutes before euthanasia to stain vessels. Plugs were harvested, frozen in liquid nitrogen, and cryosectioned. Frozen sections were fixed in cold methanol for 5 minutes and either stained with hematoxylin and fuschin stain or analyzed by immunofluorescence confocal microscopy. Microvascular density and size of the vessels were analyzed using ImageJ. Perfusion was analyzed as Alexa 488-dextran-positive vessels in relation to human CD31-positive vessels.

\section{Statistical methods}

Data are expressed as means \pm standard error of the mean (SEM). Demographic data of the PT and term populations were analyzed qualitatively using the $\chi^{2}$ test of Pearson and quantitatively using the 2-tailed unpaired $t$ test. For all other data, statistical significance was assessed by an unpaired Student $t$ test, and comparison among multiple groups by analysis of variance with the Bonferroni post hoc test. Normality was confirmed with a D'Agostino and Pearson omnibus test. For data not normally distributed, we used a Mann-Whitney test. A $P$ value $<.05$ was considered as statistically significant. Data were analyzed with GraphPad Prism software.

\section{Results}

\section{PT birth accelerates ECFC senescence}

We previously described impaired clonogenic and angiogenic activity of PT-ECFCs. ${ }^{17}$ To determine whether these defects could result from cellular senescence, we extended this study on the samples used in the previous study, ${ }^{17}$ but also by including new patients (supplemental Table 1) to further investigate the mechanisms sustaining our previous observations. We first confirmed the absence of apoptosis detected by Annexin-V/7-AAD staining in 
A

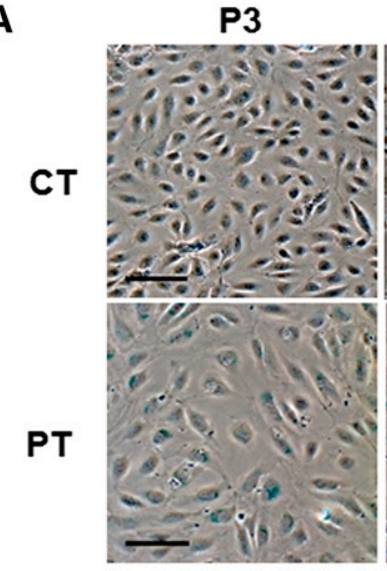

P6

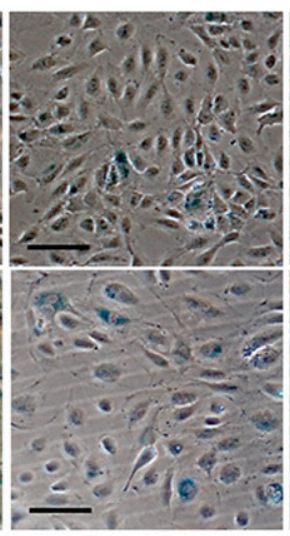

P8

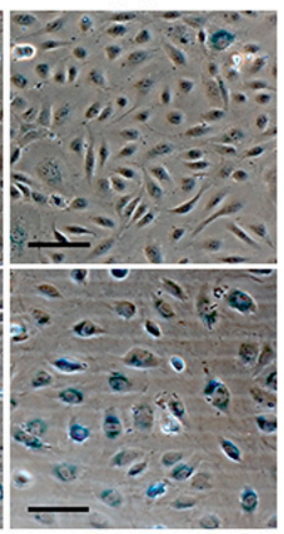

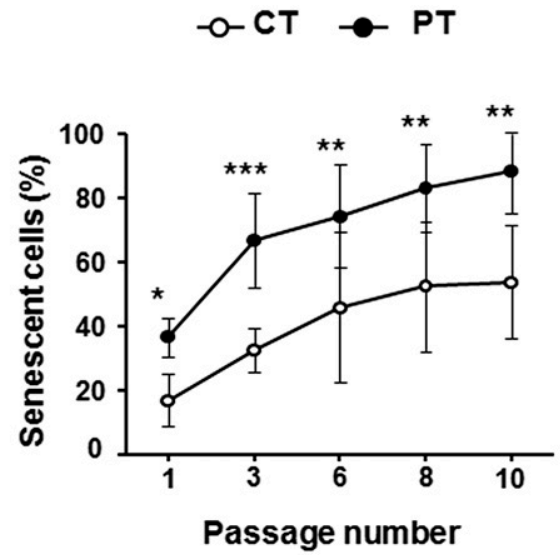

B

C

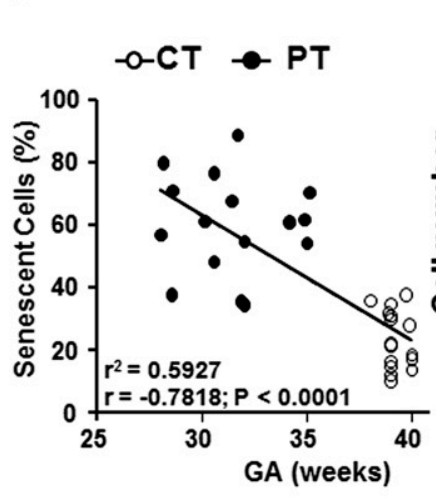

CT

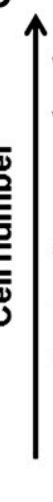

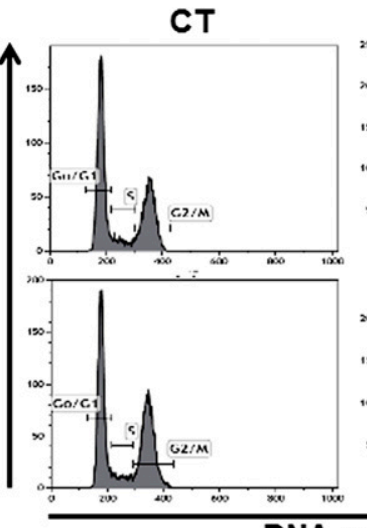
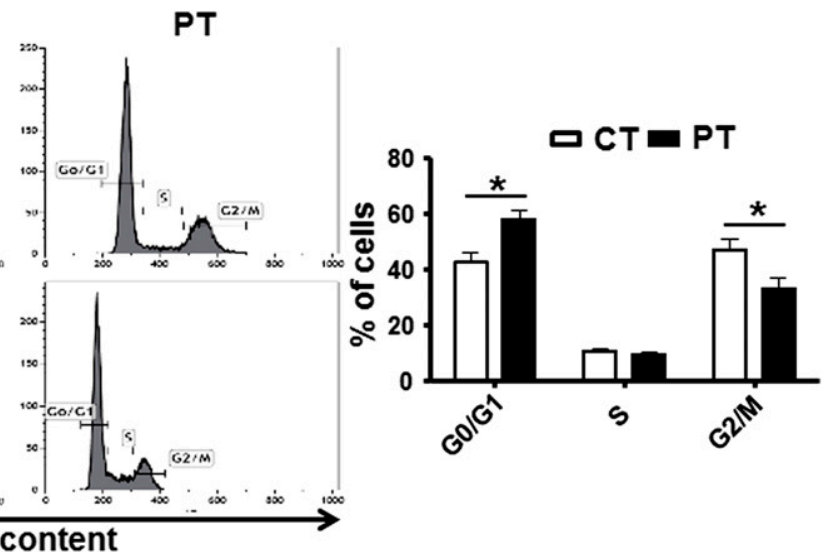

Figure 1. PT birth accelerates cellular senescence and proliferative arrest of ECFCs. SA- $\beta$-gal activity and cell-cycle analyses were assessed as markers of cellular senescence. (A) Representative images from CT- and PT-ECFCs at passage 3 (P3), 6 (P6), and 8 (P8) are shown in left panel (magnification $\times 20$; scale bar, 100 $\mu$ m). The percentage of senescent cells in PT-ECFCs was determined as the number of cells expressing SA- $\beta$-gal (blue cells) relative to the total number of cells in each field in PT neonates $(n=16)$ and CT neonates $(n=16)$. Graph (right panel) represents means \pm SEM of 16 independent samples in continuous passage assay. Experiments were done in triplicate, ${ }^{\star} P<.05 ;{ }^{\star \star} P<.01 ;{ }^{\star \star \star} P<.001$. (B) SA- $\beta$-gal activity negatively correlated with gestational age in samples from all groups ( $\mathrm{n}=32$, $\mathrm{P} 3$ ); $\mathrm{r} 2$, correlation coefficient of determination; r, Pearson correlation coefficient. (C) Cell-cycle analysis by flow cytometry. The DNA content in each cell-cycle phase in CT- and PT-ECFCs were analyzed by flow cytometry after propidium iodide staining. The representative histograms are from 2 samples per groups (left panel). Graph (right panel) represents a mean percentage of cells at different phases of the cell cycle determined by the DNA content \pm SEM for 5 to 8 independent samples. ${ }^{\star} P<.05$.

PT-ECFCs compared with CT-ECFCs (supplemental Figure 1A). After plating, we observed a significant decrease in the growing capacity of PT-ECFCs compared with controls (supplemental Figure 1B). This reduced growing capacity was associated with morphological changes and appearance of enlarged flattened cells in PT-ECFCs (Figure 1A). Using SA- $\beta$-gal staining, we found that PT-ECFCs were highly senescent compared with controls, with senescence observed at very early passages (Figure 1A) while up to $80 \%$ and $100 \%$ of senescent cells were respectively observed at passage 6 and 8. Interestingly, senescence was inversely correlated with gestational age (Figure 1B). To confirm senescence, analysis of cell-cycle distribution by flow cytometry revealed a drastic cell-cycle arrest at the G0/G1 phase (Figure 1C, supplemental Figure 1C) We infer from these results that PT reduces the growing capacity of ECFCs by inducing an early senescence.

\section{PT-ECFC aging is associated to stress-induced premature senescence}

To investigate the cellular and molecular defects underlying PT-ECFC senescence, we first measured telomere length by telomere restriction fragment. Telomeres were not shortened in PT-ECFCs compared with CT-ECFCs (supplemental Figure 2). Moreover, PTECFCs did not show any increase in $\gamma \mathrm{H} 2 \mathrm{AX}$-DNA damageinduced foci (data not shown).
The major pathways controlling senescence converge at the level of activation of the cyclin-dependent kinase inhibitor $\mathrm{p} 16^{I N K 4 a}$ or $\mathrm{p} 21^{W A F}$ keeping the pRb protein in its active form. ${ }^{19}$ Therefore, we assessed the expression of several key cell-cycle markers by quantitative RT-PCR and immunoblotting. Among them, p53 level was not modulated (Figure 2A left, B), p2 $1^{W A F}$ was significantly decreased (Figure $2 \mathrm{~A}$ middle, C) while p16 ${ }^{I N K 4 a}$ was significantly upregulated both at the RNA and protein levels in PT-ECFCs compared with CT-ECFCs (Figure 2A right, D). Furthermore, we assessed whether phosphorylation of the $\mathrm{Rb}$ tumor suppressor, a well-known cell-cycle regulator, was modulated in senescent PT-ECFCs. By comparing the ratio of intensity between $\mathrm{P}-\mathrm{Rb} / \mathrm{Rb}$, we found that the $\mathrm{Rb} 2$ hypophosphorylated form was significantly reduced independent of $\mathrm{Rb}$ loss (Figure 2E). Altogether, $p 16^{I N K 4 a} / \mathrm{Rb}$ pathway activation in the absence of $\mathrm{p} 53 / \mathrm{p} 21^{\text {WAF }}$ pathway modulation, telomere shortening, and DNA damage response indicates that premature aging in PT-ECFCs does not result from replicative senescence but is rather associated with stress-induced premature senescence. ${ }^{20-22}$

\section{PT-ECFC senescence is associated with changes in SIRT1 expression}

We then investigated whether modulation of the SIRT proteins, considered as regulators of longevity or aging in different organisms, 
A
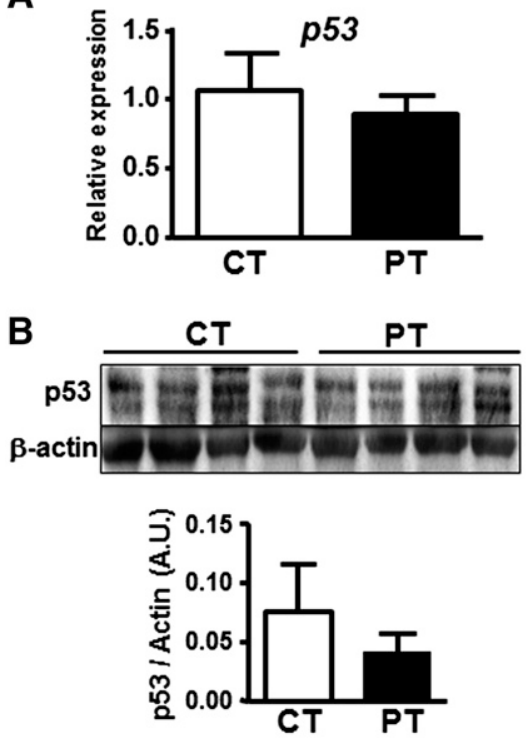

E

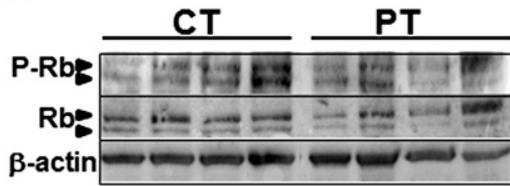

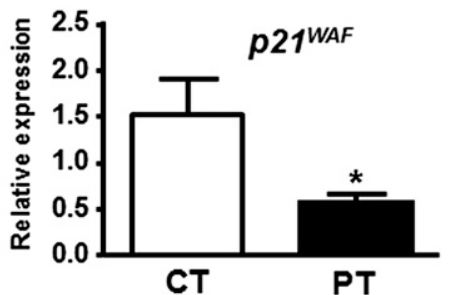

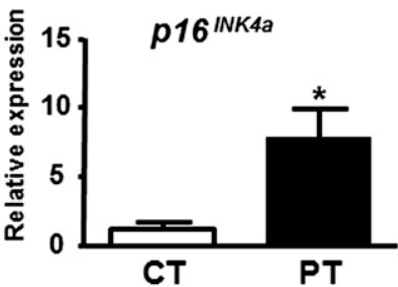

C
p21
B-acti
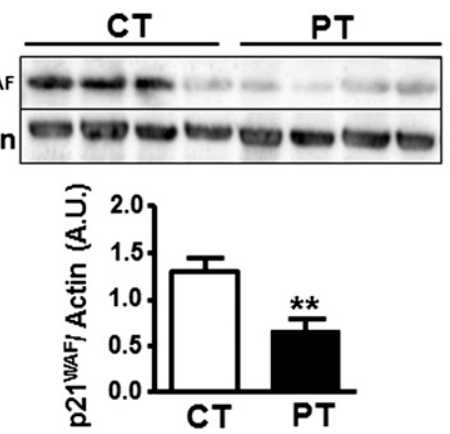
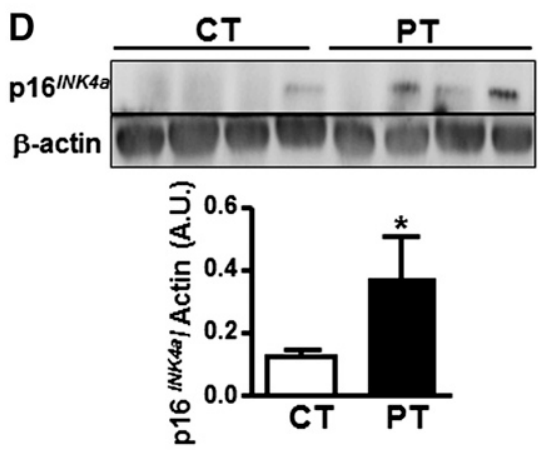
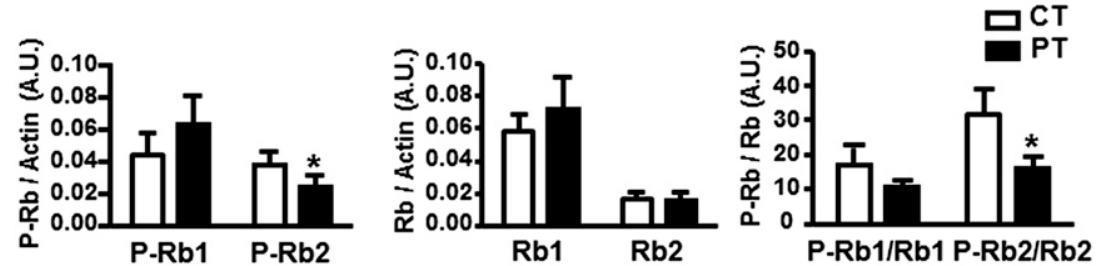

Figure 2. Senescence of PT-ECFCs is associated with changes in expression for cell-cycle checkpoint factors and Rb phosphorylation. p53, p2 $1^{\text {WAF }}$, and $\mathrm{p} 16{ }^{\mathrm{INK} 4 a}$ expression and Rb phosphorylation were assessed as senescence markers. (A) Relative mRNA expression of different cell-cycle markers was determined by quantitative RT-PCR analysis on a Stratagen Mx3000. For each gene, data were normalized to the housekeeping gene RPL13A. Graphs are representative of 4 and 10 samples for CT and PT-ECFCs, respectively. Experiments were performed in duplicate. ${ }^{\star} P<.05$. (B-D) The level of proteins involved in the regulation of the G1 phase was examined by immunoblot on whole-cell lysates from CT- and PT-ECFCs as described in "Patients, materials, and methods." Representative experiment for p53 (B), p21 WAF (C), and p16 ${ }^{I N K 4 a}(\mathrm{D})$ in 4 independent CT and PT-ECFCs are shown in upper panel. A total of $30 \mu \mathrm{g}$ of cell lysate was loaded on $4 \%$ to $12 \%$ sodium dodecyl sulfate-polyacrylamide gel electrophoresis (SDS-PAGE) gradient under reducing conditions. Blots were probed with anti-p53, -p16 ${ }^{I N K 4 a}$, -p2 ${ }^{\text {WAF }}$ polyclonal antibodies or with anti- $\beta$-actin polyclonal antibody as loading control. Protein quantification was presented by bar graphs (lower panels) showing means \pm SEM of densitometry ratio for each protein normalized to $\beta$-actin of 4 independent immunoblots. ${ }^{\star} P<.05 ;{ }^{\star} P<.01$. (E) Changes in the phosphorylation state of Rb were analyzed by immunoblots using antibody against total (Rb) and phosphorylated $\mathrm{Rb}(\mathrm{P}-\mathrm{Rb})$. Representative experiment is shown. Histograms display normalized staining intensity measured by densitometry. Left, $\mathrm{P}-\mathrm{Rb}$ to $\beta$-actin ratio; middle, $\mathrm{Rb}$ to $\beta$-actin ratio; and right, $\mathrm{P}-\mathrm{Rb}$ to $\mathrm{Rb}$ ratio. ${ }^{\star} P<.05$

was associated with the premature senescence of PT-ECFCs. We did not observe any significant variation of SIRT6 (data not shown) while a significant decrease in SIRT1 both at the RNA and protein level was detected in PT-ECFCs (Figure 3A-B). Immunofluorescence confirmed that the number of SIRT1-positive nuclei was significantly lower in PT-ECFCs (Figure 3C). Consistent with this finding, the level of acetylated p53 at Lys-382, a target of SIRT1 deacetylase, was significantly higher in PT-ECFCs, suggesting overall reduced SIRT1 activity (Figure 3E). Strikingly, SIRT1 level positively correlated with gestational age (supplemental Figure 3A-B) and inversely correlated with the percentage of senescent cells (Figure 3D; supplemental Figure 3C) suggesting a connection between the level of SIRT1 and prematurity.

SIRT1 expression is subjected to several levels of control. Among them, we tested whether epigenetic changes might contribute to silencing of the SIRT1 gene. Regulation of SIRT1 expression was not associated with hypermethylation of the gene promoter region (supplemental Figure 4A-D). Furthermore, chromatin immunoprecipitation followed by quantitative PCR revealed that the "active" marks trimethyl-H3K4 (H3K4me3) associated with the SIRT1 promoter were significantly decreased in PT-ECFCs compared to CT-ECFCs, whereas the repressive marks trimethylH3K9, associated with heterochromatin formation, ${ }^{23}$ was increased (supplemental Figure 4E). These results demonstrate that
SIRT1 repression in PT depends at least in part, on a loss of "active" epigenetic marks at its promoter.

To test whether SIRT1 deficiency occurs in vivo and is not a consequence of ECFCs culture, we measured SIRT1 expression in cord blood MNCs. SIRT1 expression was also decreased in MNCs from PT compared with CT neonates (Figure 3F), indicating that SIRT1 deficiency is not restricted to ECFCs but is also detectable in circulating blood cells.

\section{SIRT1 level drives PT-ECFC accelerated senescence}

To uncover the causal relationships between accelerated senescence and SIRT1 downregulation in PT cells, we transfected PT-ECFCs either with a SIRT1 or empty vector. Twenty-four hours after transient transfection, SIRT1 overexpression (Figure 4A-B upper panel) was associated with a significant decrease in the level of the senescence-associated $\mathrm{p} 16^{I N K 4 a}$ protein (Figure 4A-B middle panel), whereas $\mathrm{p} 21^{W A F}$ was not significantly modulated (Figure 4A-B bottom panel). Consistently, transfection of PT-ECFCs with the SIRT1 vector significantly reduced SA- $\beta$-gal activity and senescenceassociated morphological changes, compared with PT-ECFCs transfected with an empty vector (Figure 4C). Interestingly, addition of NAM, a competitive inhibitor of SIRT1 activity, ${ }^{24,25}$ counteracted the beneficial effect of SIRT1 overexpression on the 
A

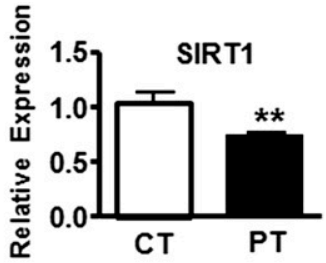

C
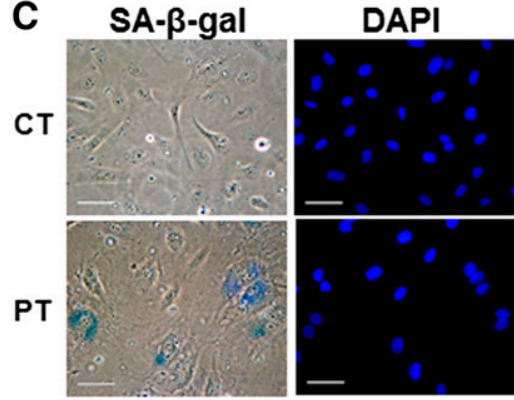

E

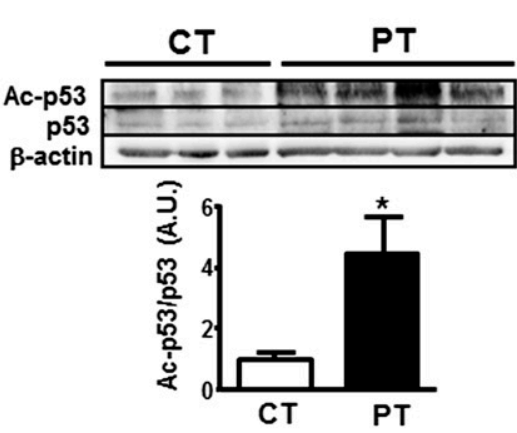

B

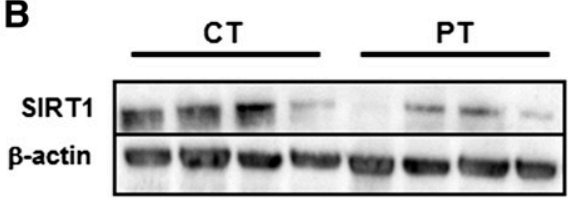

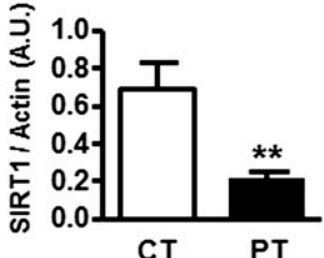

D

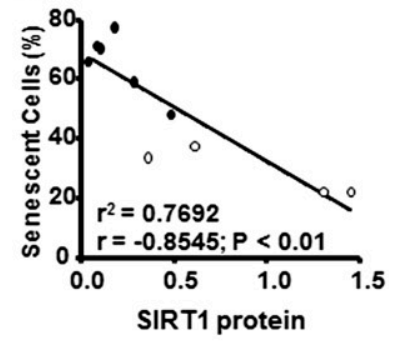

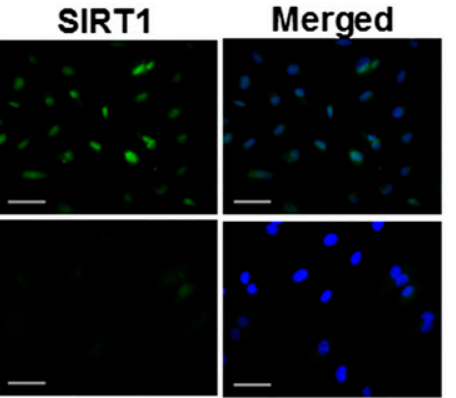

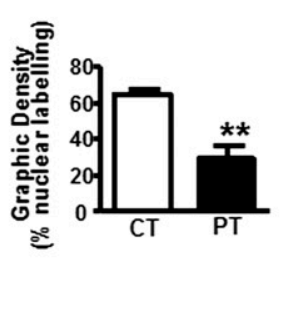

F

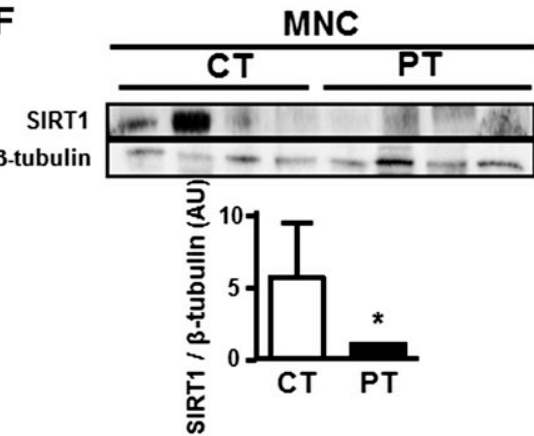

Figure 3. Accelerated senescence in PT-ECFCs is correlated to decreased SIRT1 levels associated to reducing activity. SIRT1 expression was assessed in ECFCs from CT and PT neonates at the RNA and protein level. (A) Relative SIRT1 expression was determined by quantitative RT-PCR analysis on a Stratagen Mx3000. Data were normalized to the housekeeping gene $R P L 13 A$. Graph is representative of 7 and 12 experiments performed in duplicated for CT- and PT-ECFCs, respectively. ${ }^{\star} P<.01$. (B) Western blot analysis of SIRT1 from ECFCs of 4 representative CT and PT neonates was shown. Histograms display mean \pm SEM of SIRT1 intensity measured by densitometry normalized to $\beta$-actin for 4 independent experiments. ${ }^{\star \star} P<.01$. (C) Costaining between SA- $\beta$-gal activity and SIRT1 protein were realized as described in "Patients, materials, and methods." CT- and PT-EFCFs were stained by immunofluorescence for SIRT1 protein (green). Nuclei were counterstained with 4,6 diamidino-2phenylindole (DAPI) (blue). Representative images from the 2 groups are shown (original magnification $\times 20$; scale bar, $50 \mu \mathrm{m}$ ). Bars represent means \pm SEM of the number of cells with a SIRT1 nuclear signal relative to the total cell number. ${ }^{* \star} P<.01$. (D) SA- $\beta$-gal activity negatively correlated with SIRT1 protein level in samples from all groups $(n=10, P 3)$; 2 , correlation coefficient of determination; $r$, Pearson correlation coefficient. (E) Changes in the acetylation state of p53 were analyzed by immunoblots using antibody against total and acetyl p53. Representative experiments is shown. Bars display densitometry analysis after normalization to $\beta$-actin as mean \pm SEM of the relative intensities of 4 independent experiments. ${ }^{*} P<.05$. (F) Western blot analysis of SIRT1 from MNC of 4 representative CT and PT neonates was shown. Bars display densitometry analysis of SIRT1 after normalization to $\beta$-tubulin as mean \pm SEM of the relative intensities of 2 independent experiments. ${ }^{\star} P<.05$.

senescent phenotype of PT-ECFCs (Figure 4C) and was associated with upregulation of $\mathrm{p} 16^{I N K 4 a}$ expression while SIRT1 expression was not modified (supplemental Figure 5A-B). Consistent with the reduction of SIRT1 in senescent PT-ECFCs, SIRT1 overexpression accelerated PT-ECFC proliferation, an effect blocked by NAM (Figure 4D). Altogether, these data indicate that SIRT1 level drives ECFCs accelerated senescence in PT neonates.

\section{RSV reverses senescence and improves in vitro angiogenesis of PT-ECFCs in a SIRT1-dependent manner}

Given the role of SIRT1 deficiency on PT-ECFC senescence, we tested whether RSV may postpone senescence onset. A time-course assay was carried out on PT-ECFCs to investigate the impact of $1 \mu \mathrm{M}$ RSV on SIRT1 and senescence-associated markers. RSV increased SIRT1 gene expression in a time-dependent manner (Figure 5A) which correlated with accumulation of active chromatin marks at the SIRT1 promoter (Figure 5B). Moreover, RSV globally increased SIRT1 cellular (Figure 5C) and nuclear (Figure 5D) levels and induced a gradual decrease in $16^{I N K 4 a}$ expression (Figure 5C) in time-dependent manner, whereas the level of $\mathrm{p} 21^{W A F}$ level was unchanged (Figure 5C). Accordingly, RSV treatment significantly reduced the percentage of senescent cells to a level similar to CTECFCs, while this effect was counteracted by NAM (Figure 5E; supplemental Figure 6). We conclude that RSV rescues ECFCs senescent phenotype in a SIRT1-depedent manner.

Having demonstrated that RSV prevents senescence onset, we examined whether the drug would increase proliferation and counteract the impaired angiogenic capacity of PT-ECFCs. RSV significantly increased the mitotic potential of PT-ECFCs, as shown by BrdU incorporation assay, an effect reverted in the presence of NAM (Figure 6A). By using immunofluorescent staining, we found that RSV treatment of PT-ECFCs postponed the G0/G1 arrest (supplemental Figure 7A) and concluded that RSV treatment rescues cell proliferation capacity by increasing the G1/S transition.

In addition, in Matrigel assays, RSV treatment significantly increased endothelial network formation by PT-ECFCs, as shown by increased number of closed tubes and branches (Figure 6B-C). 
Figure 4. SIRT1 overexpression after transient transfection decreases senescence and restores cell proliferation. PT-ECFCs were untransfected (Mock), transiently transfected with pCMV-sport6 vector (Empty) or the pCMV-Sport6-SIRT1vector (SIRT1), and treated or not with NAM (1mM). (A) Transient overexpression of SIRT1 was monitored by western blot (upper panel) together with expression of p $16^{\text {INK4a }}$ and p21 ${ }^{\text {WAF }}$. After 24 or 48 hours, cells were lysed and $30 \mu \mathrm{g}$ of lysates were resolved on $4 \%$ to $12 \%$ SDS-PAGE gradient under reducing conditions. Blots were probed with anti-SIRT1,-p16 INK4a, $-p 21^{\text {WAF }}$ polyclonal antibodies or with anti- $\beta$-actin polyclonal antibody as a loading control. (B) Fold induction of SIRT1, p16 INK4a, and p21 WAF expression in SIRT1-transfected PT-ECFCs compared with cells transfected with the empty vector at the same time point. Bars represent mean \pm SEM of the relative intensities of 8 independents experiments. ${ }^{\star} P<.05$; ${ }^{* *} P<.01 ;{ }^{* * *} P<.001$. (C) Two days after transfection, SA- $\beta$-gal staining was performed for evaluation of the senescent status (left panels). A representative image of CT-ECFCs is shown. Other images correspond to PT-ECFCs after different treatments (original magnification $\times 20$; scale bar, $100 \mu \mathrm{m})$. SA- $\beta$-gal-positive cells were counted and presented as percentage of senescent cells per total cell number (Right panel). Data are means \pm SEM of 12 independents samples. For each, experiments were carried in triplicate. ${ }^{*} P<.05$; ${ }^{\star \star} P<.01$. (D) Proliferation rate was determined by BrdU incorporation assay after transient transfection in 7500 ECFCs. Results were expressed as arbitrary units of spectrophotometric measurements. Data are means \pm SEM of 12 independent samples. For each, experiments were performed in triplicate. ${ }^{\star} P<.05 ;{ }^{\star \star} P<.01$.
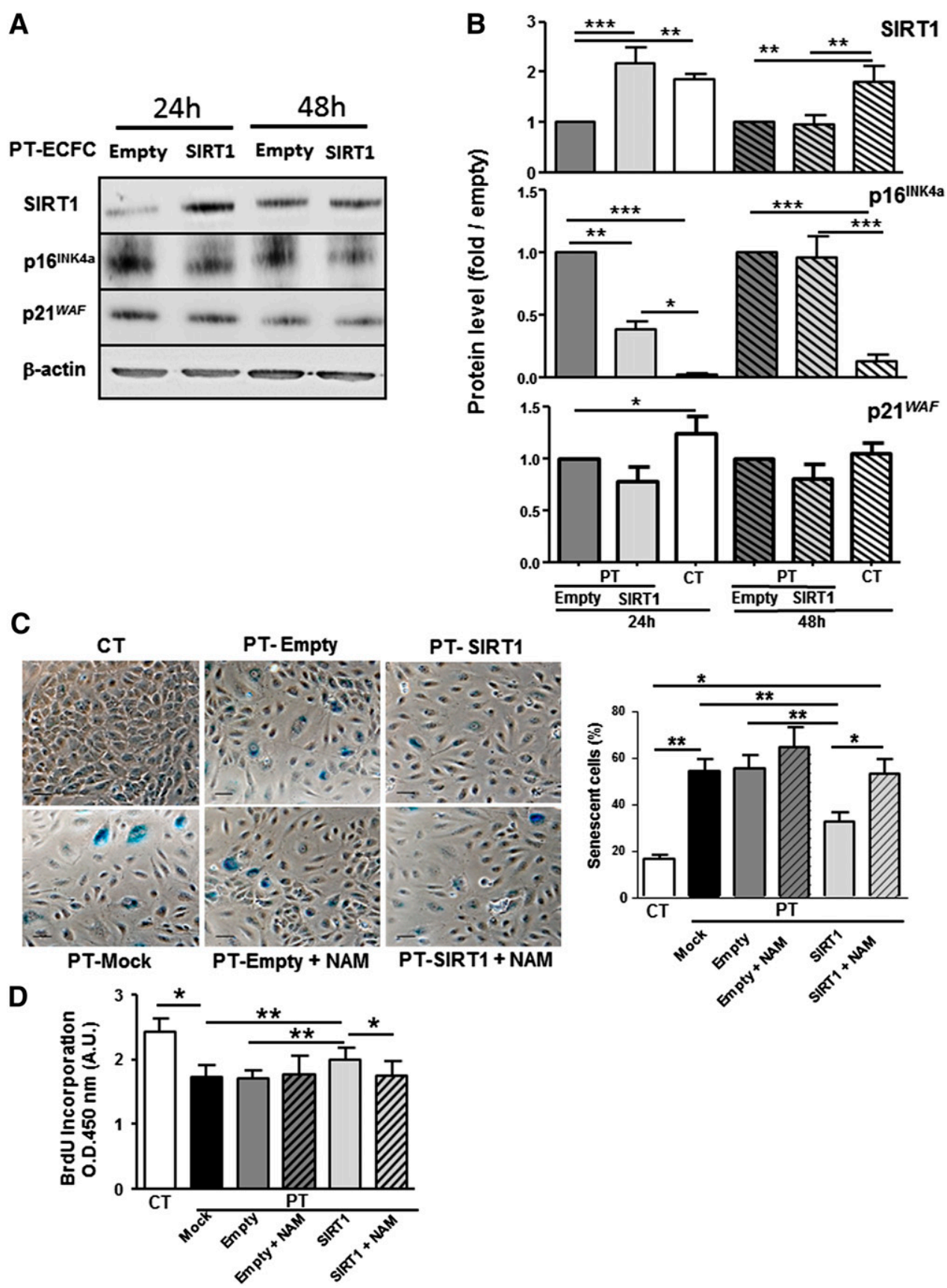

PT- SIRT1
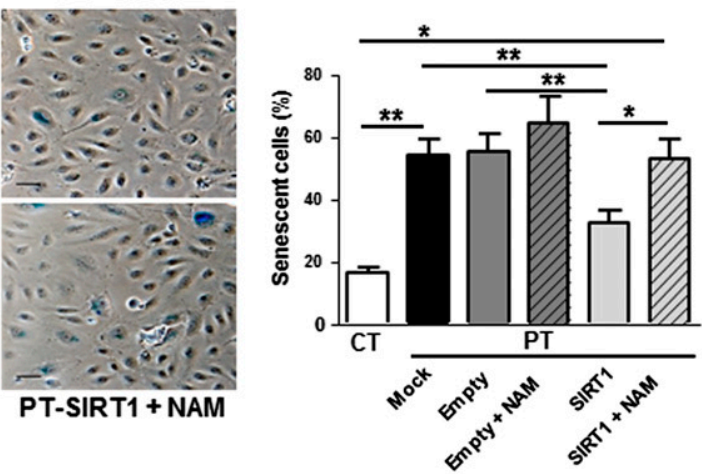

Moreover, in a three-dimensional spheroid assay, sprouts formation, length, and branching were improved by RSV (Figure 6D-G), revealing a significant effect of the drug in restoring the in vitro angiogenic properties of PT-ECFCs. On the opposite, inhibition of SIRT1 activity by addition of NAM to RSV-treated cells abrogated the beneficial impact of the drug on tube (Figure 6B-C) and sprout formation (Figure 6D-G). To confirm this SIRT1-mediated regulation of PT-ECFC angiogenic dysfunction, spheroid assays were performed on SIRT1 or empty vector-transfected cells. Sprouts and branch number as well as cumulative length were significantly increased in SIRT1-transfected PT-ECFCs while the effect of SIRT1 overexpression was abrogated by NAM (supplemental Figure 7B-E).

Interestingly, we previously identified TSP1 as involved in PT-ECFC angiogenic defects. ${ }^{17}$ We therefore investigated the impact of SIRT1 on TSP1 expression. Transient SIRT1 overexpression or
RSV treatment significantly decreased TSP1 expression (supplemental Figure 8). Overall, we conclude that RSV rescues the senescent phenotype and in vitro angiogenic deficiency of PT-ECFCs in a SIRT1dependent manner. This effect may involve TSP1 modulation.

\section{Impaired angiogenic capacity of PT-ECFCs is restored by RSV in vivo}

We next asked whether RSV treatment might also restore the angiogenic potential of PT-ECFCs in vivo. Matrigel plug assays were performed in nu/nu mice by using CT-ECFCs or PT-ECFCs embedded in Matrigel in the presence of either DMSO or RSV. As controls, Matrigel implants that did not contain ECFCs ("no cells" plugs) and supplemented with DMSO (Figure 7Ai) or RSV (Figure 7Aii) were devoid of vessels. Histological staining of the vessel wall and 


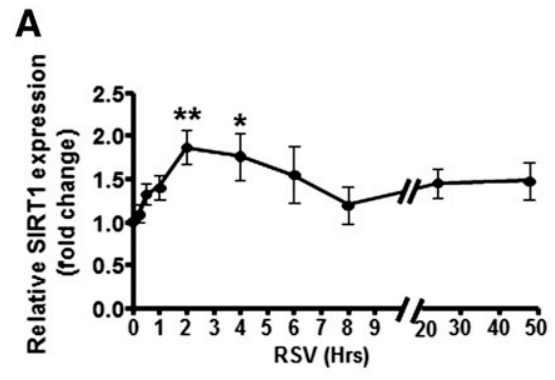

C
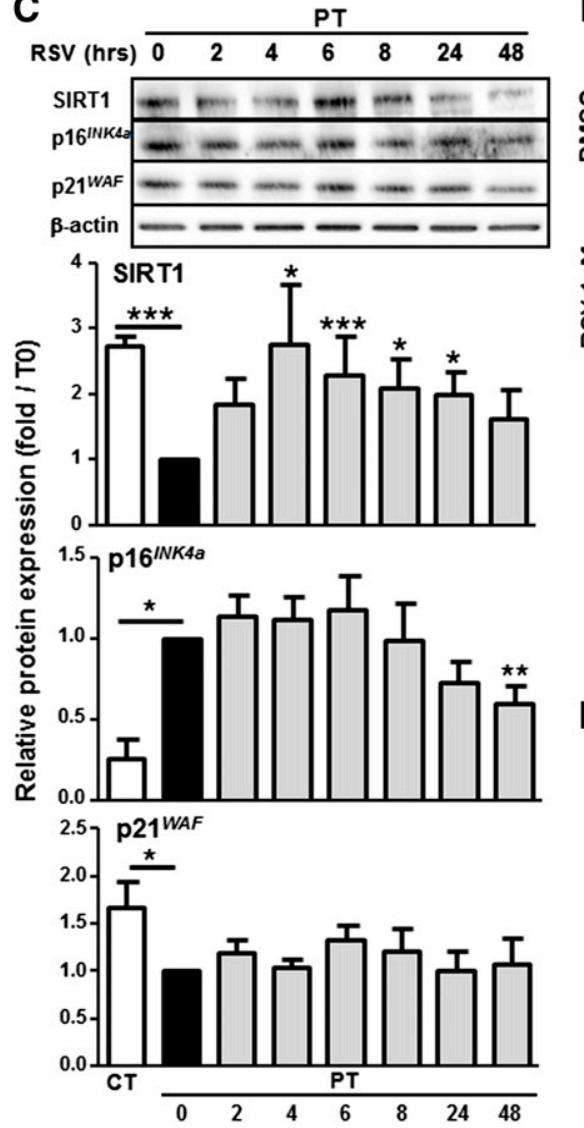

B

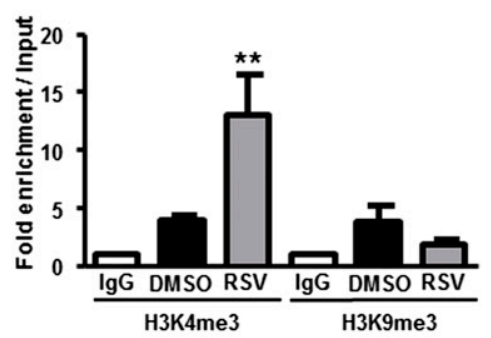

D
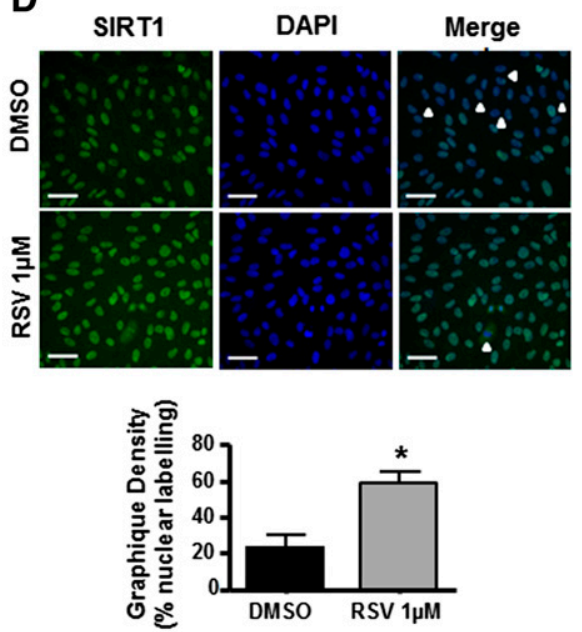

E

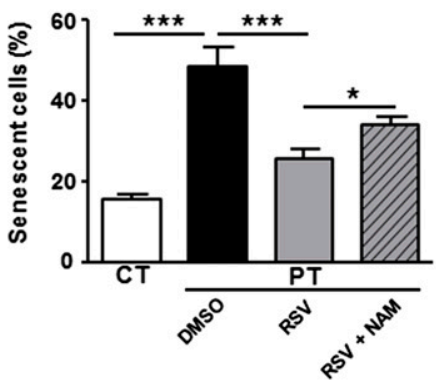

Figure 5. RSV reverts senescence by increasing SIRT1 expression. PT-ECFCs were treated with solvent alone (DMSO) or $1 \mu \mathrm{M}$ RSV for different times. (A) Time-dependent changes in the level of SIRT1 were determined by quantitative RT-PCR analysis on a Stratagen MX3000. Changes in SIRT1 mRNA were normalized to the housekeeping gene RPL13A, as described in "Patients, materials, and methods." Results were expressed as fold induction of SIRT1 mRNA level in RSV-stimulated PT-ECFCs compared with DMSO-treated PT-ECFCs in 10 independent samples. Data are means \pm SEM, ${ }^{\star} P<.05 ;{ }^{\star \star} P<$ .01. (B) RSV- and DMSO-treated PT-ECFCs were analyzed by chromatin immunoprecipitation assays. Relative enrichment of 2 chromatin marks, H3K4me3 (active chromatin) and $\mathrm{H} 3 \mathrm{~K} 9 \mathrm{me} 3$ (repressed chromatin) at the SIRT1 promoter was determined in PTECFCs by quantitative PCR after 48 hours of treatment and calculated as the ratio between bound sample and the input DNA (total DNA after crosslinking). Data are means \pm SEM of 6 independent samples. Experiments were performed in triplicate. ${ }^{\star *} P<.01$. IgG, immunoglobulin G. (C) Time-dependent modulation in SIRT1, p16 ${ }^{\text {INK4a }}$, and p21 WAF proteins in RSV-treated PTECFCs. Each whole-cell lysate $(30 \mu \mathrm{g})$ was resolved on $4 \%$ to $12 \%$ SDS-PAGE gradient under reducing conditions. Blots were probed with anti-SIRT1, p16 ${ }^{\text {INK4a }}$ p21 ${ }^{\text {WAF }}$ polyclonal antibodies or with anti- $\beta$-actin polyclonal antibody as a loading control. Staining intensity was measured by densitometry and normalized to $\beta$-actin. Results were expressed as fold induction of protein expression in RSV-stimulated PT-ECFCs compared with DMSO-treated PT-ECFCs. Bars represent mean \pm SEM of the relative intensities in 12 independents samples. ${ }^{\star} P<.05 ;{ }^{\star \star} P<.01 ;{ }^{\star \star \star} P<.001$. (D) Immunofluorescence staining of RSV-treated or not PT-EFCFs after 48 hours for SIRT1 (green, left panels). Nuclei were counterstained with DAPI (blue). Representative images are shown from the 2 groups (original magnification $\times 20$; scale bar, $50 \mu \mathrm{m})$. White arrows indicate low SIRT1 staining. Bars represent means \pm SEM of the number of cells with SIRT1 nuclear staining relative to the total cell number. ${ }^{*} P<.05$. (E) After 48 hours of DMSO or RSV treatment, in the presence or absence of $1 \mathrm{mM}$ NAM, SA- $\beta$-gal staining of PT-ECFCs was performed for evaluation of the senescence status. The SA- $\beta$-gal-positive cells were counted and presented as percentage of the total cells. Data are means \pm SEM of 10 independents samples. For each, experiments were carried in triplicate. ${ }^{\star} P<.05$; ${ }^{* \star *} P<.001$. immunofluorescent staining of vascular markers evidenced the impaired capacity of PT-ECFC to form blood vessels compared with CT-ECFCs (Figure 7Aiii-iv vs Av-vi, Ba-c vs Bd-f, C). In implants containing PT-ECFCs, RSV treatment did not affect the overall microvasculature density (Figure 7C), but significantly increased the number of large capillary structures and improved perfusion compared with untreated PT-ECFCs (Figure 7Aiii-iv vs Avii-viii, Bd-f vs Bg-i. D-E). Furthermore, in presence of RSV, the average diameter of hCD31-positive vessels formed by PT-ECFCs was significantly higher compared with untreated PT-ECFCs (Figure 7Aiii-iv vs Avii-viii, Bd-f vs $\mathrm{Bg}-\mathrm{i}, \mathrm{F})$. Taken together, RSV has a strong angiogenic effect on PT-ECFCs in vivo, mostly improving the perfusion functionality and mature vessels formation rather than the number of vessels formed.

\section{Discussion}

Epidemiologic studies have evidenced a strong association between PT birth and the development of cardiovascular disease at adulthood, possibly linked to antenatal or perinatal adverse events. However, the cellular and molecular mechanisms underlying this association remain poorly understood. Here, we demonstrate for the first time that prematurity is associated with SIRT1 deficiency that accelerates the onset of ECFC senescence and acts as a critical determinant of ECFCs angiogenic defect.

Senescence of endothelial progenitor cells limiting the ability for angiogenesis and vascular healing ${ }^{8,26,27}$ has emerged as a contributor to endothelial dysfunction developing under cardiovascular risk factors. ${ }^{20}$ This study shows that accelerated senescence in PT-ECFCs is inversely correlated with gestational age, suggesting a key role in the pathophysiological mechanisms driving early vascular dysfunction in adults born prematurely.

We found that PT-associated senescence is not dependent on telomere shortening or activation of the $\mathrm{p} 53 / \mathrm{p} 21^{\text {WAF }}$ pathway. Increased expression of $\mathrm{p} 16^{\mathrm{INK} 4 \mathrm{a}}$ together with reduced $\mathrm{pRB}$ phosphorylation blocking the entry of proliferating cells into $S$ phase indicate that PT-ECFC aging results from stress-induced premature senescence rather than replicative senescence. ${ }^{20,21}$ This senescence 
A

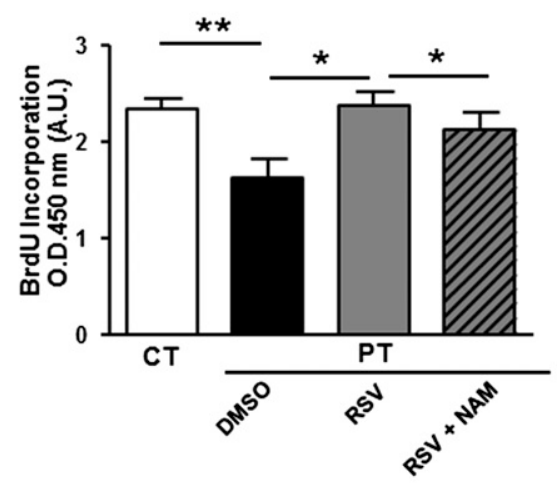

D
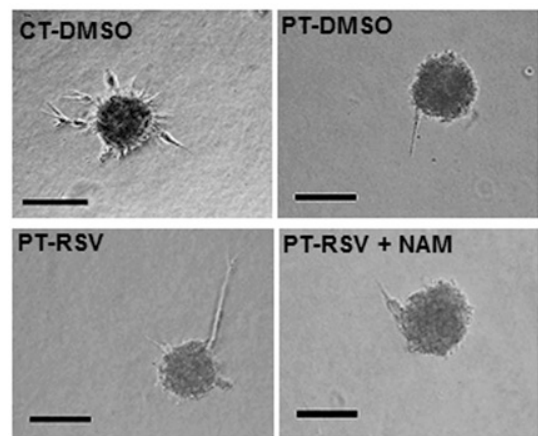

B

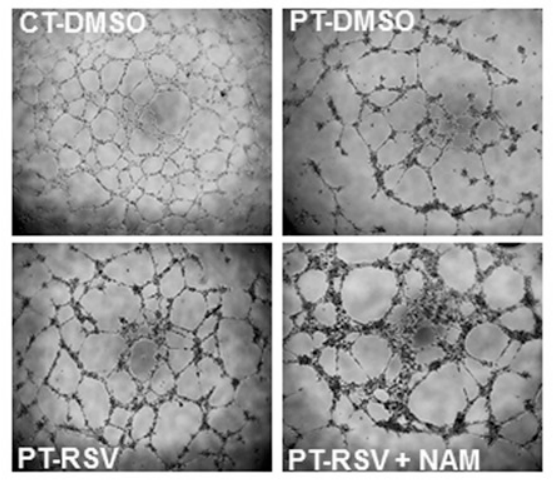

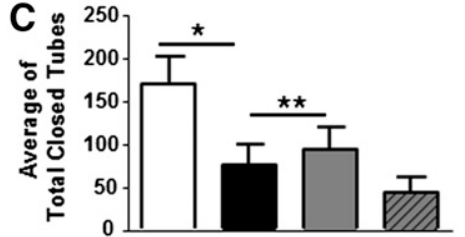

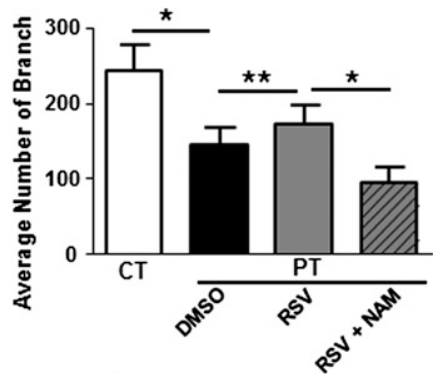

G

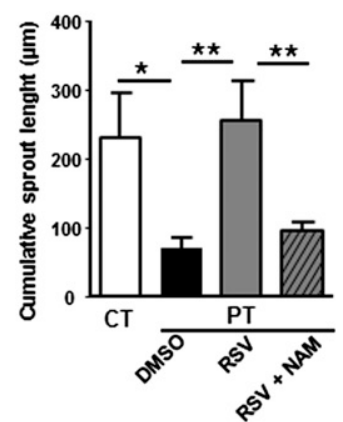

Figure 6. RSV restores proliferation and angiogenesis of PT-ECFC in vitro in a SIRT1-dependent manner. ECFCs were used at passage 3 . (A) Proliferation rate determined by BrdU incorporation assay in 7500 ECFCs after 72 hours of treatment with $1 \mu \mathrm{M}$ RSV. Results were expressed as arbitrary units of spectrophotometric measurements. Data are means \pm SEM of 12 independent samples in experiments performed in triplicate. ${ }^{\star} P<.05$, ${ }^{\star \star} P<.01$. (B) Representative experiment of capillary tube formation by ECFCs from CT or PT-neonates treated with $1 \mu \mathrm{M}$ RSV, in the presence or not of $1 \mathrm{mM}$ NAM. A total of 20000 ECFCs per well were seeded on growth factor-reduced Matrigel. Tube formation was analyzed 6 hours later on a phase-contrast microscope (original magnification $\times 4$ ). (C) Quantitative analysis of total closed tubes (top panel) and branches (bottom panel) after 6 hours of treatment. Data are means \pm SEM of 12 independent samples in experiments performed in triplicate. ${ }^{\star} P<.05$, ${ }^{\star \star} P<.01$. (D) Representative experiment of three-dimensional in vitro angiogenesis assay with collagen gel-embedded spheroids of PT-ECFCs treated with RSV (1 $\left.\mu \mathrm{M}\right)$, in the presence or not of $1 \mathrm{mM}$ NAM and CT-ECFCs (original magnification $\times 20$; scale bars, $100 \mu \mathrm{m}$ ). (E) Quantification of the number of sprouts per spheroid. (F) Quantification of the number of branch points per spheroid. (G) Quantification of the cumulative sprout length per spheroid. (E-G) For each experiment, sprouts from 20 spheroids were counted. Data are means \pm SEM of 12 independent samples from experiments performed in duplicate. ${ }^{\star} P<.05 ;{ }^{\star \star} P<.01 ;{ }^{\star \star \star} P<.001$.

phenotype involving $\mathrm{p} 16^{\mathrm{INK} 4 \mathrm{a}}$ without significant increase in $\mathrm{p} 21$ is consistent with previous observations in endothelial cells exposed to oxidative stress $^{28}$ and the possible independent role of these 2 pathways in senescence induction. ${ }^{22}$ Interestingly, in healthy elderly, $p 16^{I N K 4 a}$ expression was reported to increase with age and contribute to the age-dependent progenitor cell senescence. ${ }^{29}$ Our findings suggest that a common mechanism links physiological aging and accelerated senescence of PT-ECFCs.

Regulation of aging has been associated with calorie restriction and upregulation of different sirtuins. ${ }^{30}$ In human cells, 7 members of this family have been described ${ }^{31}$ and 2 of the 3 nuclear sirtuins, SIRT1 and SIRT6, contribute to longevity. ${ }^{32,33} \mathrm{We}$ demonstrate that accelerated senescence of PT-ECFCs is driven by SIRT1 levels which positive correlates with gestational age, whereas SIRT6 is not affected. The SIRT1 modulation experiments in PT-ECFCs argue in favor of a primary role of SIRT1 deficiency over $p 16^{I N K 4}$ dysregulation. Indeed, transfection-induced SIRT1 overexpression induces a significant reduction of $\mathrm{p} 16^{I N K A 4 a}$, an effect abrogated by NAM. In addition, in PT-EFCs treated with RSV, peak levels of SIRT1 protein occurred earlier than $\mathrm{p} 16^{I N K A 4 a}$ repression. Together with other models, these data suggest that environmental stress in the perinatal period might modulate senescence and $p 16^{I N K 4 a}$ expression through SIRT1-dependent pathways. ${ }^{34}$ In our study, PT-ECFC SIRT1 expression is reduced subsequent to epigenetic silencing of its promoter, suggesting a transcriptional regulation of SIRT1 rather than a mechanism downstream of SIRT1. In RSV-treated PT-ECFCs, we were not able to conclude to a FOXO1-dependent activation of SIRT1 (data not shown). However, with various transcriptional factors being reported to bind to SIRT1 promoter, ${ }^{35}$ further investigations would be required to elucidate SIRT-1 regulation in the context of prematurity.

Our study demonstrates the critical involvement of SIRT1 in the functional defect of PT-ECFCs. This observation is in line with the major role of SIRT1 in angiogenesis previously reported in mice and zebrafish models. ${ }^{36}$ Conditional deletion of SIRT1 in endothelial cells impairs angiogenic response following ischemic injury, independently of phenotypic abnormalities during embryonic development, ${ }^{36}$ highlighting the role of SIRT1 as a metabolic sensor coupling energy and oxygen homeostasis to vasculature growth. Interestingly, besides reducing cellular oxidative stress burden, SIRT1 was shown to be also highly regulated by oxidative stress. ${ }^{37,38}$ Moreover, antioxidant mechanisms are deficient in PT newborns and data are accumulating on 
A

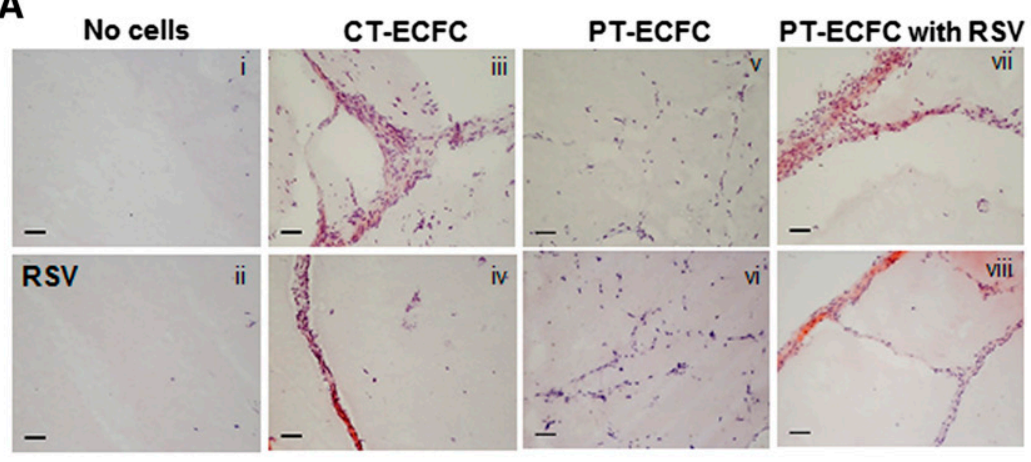

B
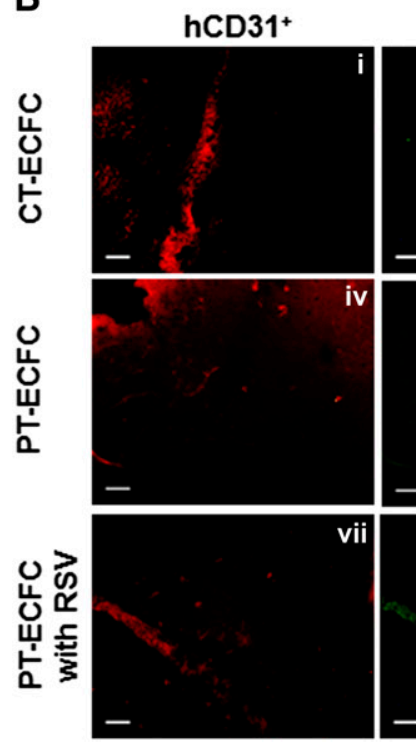
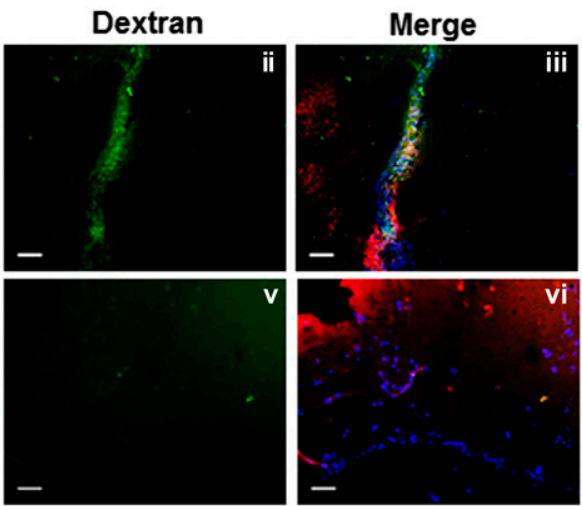

viii

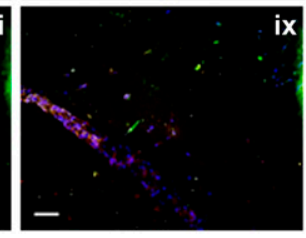

C

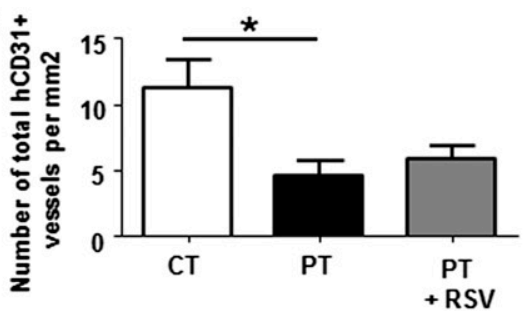

D

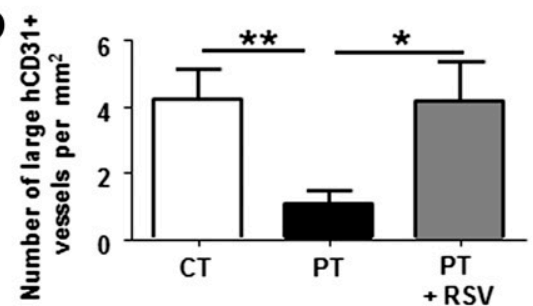

E

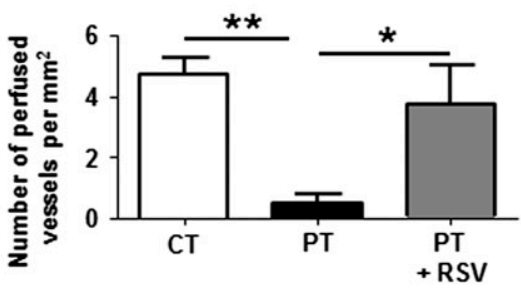

hCD31+

Dextran

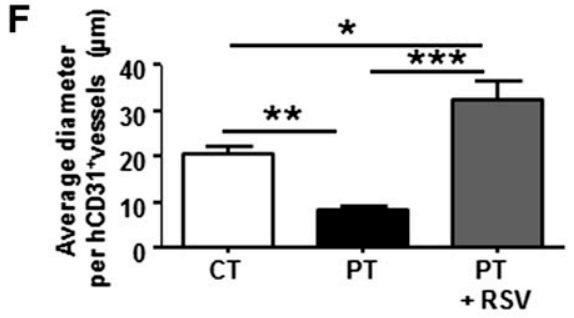

Dapi

Figure 7. RSV stimulates capacities of PT-ECFCs to form functional blood vessels in vivo. To determine the proangiogenic effect of RSV on PT-ECFCs in vivo, 2 groups of 5-week-old nu/nu mice were injected with a mixture of ECFCs and SMC (ratio 75:25) resuspended in growth factor-reduced Matrigel supplemented with either DMSO or $1 \mu$ M RSV. Implants were harvested after 7 days and stained with hematoxylin and Fuschin (A) or labeled by fluorescent staining (B) as described in "Patients, materials, and methods." (A) Representative images of luminal microvessels in sections of Matrigel plugs (original magnification $\times 20$; scale bars, $50 \mu \mathrm{m}$ ). H\&F staining showing vessel-like structures in plugs unfilled in the absence (i) or presence (ii) of $1 \mu \mathrm{M}$ RSV and filled with CT-ECFCs (iii-iv), PT-ECFCs ( $v$-vi), and PT-ECFCs treated with $1 \mu \mathrm{M}$ RSV (vii-viii). Images (v,vii) or (vi,viii) were representative of the same sample in the absence or presence of RSV. (B) Representative images of the human vasculature (shown in red by staining against hCD31) and the perfusion in the plugs (shown in green by infusion of Alexa 488-dextran). Nuclei were counterstained with DAPI (blue). Staining showing that vessel-like structures were representative of plugs filled with CT-ECFCs (i-iii), and showing PT-ECFCs in the absence (iv-vi) or presence (xii-ix) of $1 \mu \mathrm{M}$ RSV. Subpanels iv-ix were representative of the same sample in the absence or presence of RSV (original magnification $\times 20$; scale bars, $50 \mu \mathrm{m}$ ). (C) Quantification of the overall microvascular density of the human vessels per square millimeter $(n=5-7$ mice). (D) Quantification of large microvessel density per square millimeter ( $n=5-7$ mice). (E) Quantification of perfused human vessels per square millimeter, as shown by Dextran labeling $(n=5-7$ mice). ( $F)$ Quantification of the average diameter of the human vessels ( $\mathrm{n}=5-7$ mice). (C-F) Means \pm SEM from 5 nonoverlapping microscopic fields in 5 separate sections per plugs for $5-7$ plugs per groups. ${ }^{\star} P<.05 ;{ }^{\star \star} P<.01 ;{ }^{\star \star \star} P<.001$.

the role of perinatal oxidative stress as a trigger in hypertension programming. ${ }^{39}$ These data suggest that SIRT1 deficiency may be a major link between environmental oxidative stress associated to premature birth, early senescence, and dysfunction of ECFCs. Consistently, SIRT1 deficiency in adult ECFCs exposed to $\mathrm{H}_{2} \mathrm{O}_{2}$ or in ECFCs isolated from healthy smokers and COPD patients was shown to be responsible for accelerating cell aging and reducing functionality for angiogenesis. ${ }^{40}$ In that context, DNA damage could be evidenced in ECFCs, in contrast to our observation. One possible explanation for such a difference is that cells from premature neonates are analyzed at the earliest time and after standard culture conditions, and that exposure to cumulative oxidative stress is not yet sufficient for inducing DNA damage. Alterations of PT-ECFC senescence phenotype is thus likely to be a hallmark for increased susceptibility to environmental stresses that could occur later in life and contribute to poor endothelial regeneration.
Beside alteration of angiogenesis, available experimental data link SIRT1 deficiency to a wide vascular deleterious role in human. SIRT1 promotes eNOS-dependent endothelial function, ${ }^{41}$ an early marker for the development of hypertension in PT infants. ${ }^{2,3,42}$ Arterial stiffness is also regulated by SIRT1 by preventing hyperphosphathemia-induced calcification. ${ }^{43}$ Thus, alteration of the different pathways controlled by SIRT1 might contribute not only to reduction in microvascular density, but also to other vascular defects related to prematurity such as aortic dysfunction and vascular stiffening. ${ }^{2,44}$ SIRT1 also regulates the coagulation/inflammation axis ${ }^{45}$ and glucose homeostasis. ${ }^{46}$ Its downregulation has been associated with diabetes and cardiovascular diseases. ${ }^{47}$ Thus, SIRT1-dependent premature cellular aging could contribute to cardiovascular complications in adults born prematurely, mimicking thereby cardiovascular diseases in elderly linked to aging of the vascular tree. ${ }^{48,49}$ In addition, insulin resistance and metabolic syndrome, 2 of the main diseases observed at adulthood after 
a PT birth, affect SIRT1 expression in MNCs. ${ }^{46}$ The downregulation of SIRT1 that we observed in MNCs of neonates born prematurely suggests that, in circulating blood cells, SIRT1 might represent a novel marker of altered metabolism and homeostasis.

Our report also demonstrated that reversion of SIRT1 deficiency by RSV, restores angiogenic capacities of ECFCs. Of note, the beneficial effect of RSV was associated to an increased diameter of neovessels formed by PT-ECFCs in vivo. This result suggests an improvement in vessel maturation and arteriogenesis, a mechanism known to be favored by ECFCs. ${ }^{50,51}$ Our observations are consistent with the protective effects of RSV on the vascular system. ${ }^{45-48}$ Together with RSV, small-molecule activators of SIRT1 have been proposed in the treatment of type 2 diabetes, ${ }^{52,53}$ another disease associated with PT. Therefore, polyphenols might become protective molecules against premature aging in PT neonates and associated cardiovascular risk. Nevertheless, we observed that RSV differentially impacts on normal ECFCs from neonates born at term and PT-ECFCs (data not shown), as proliferation and angiogenic activity was not enhanced and even moderately decreased in controls cells. As previously reported, the angiogenesis-regulating properties of RSV are heterogeneous, and highly depend on the characteristics of target cells. ${ }^{54,55}$ The striking differences in CT-ECFCs compared with PT-ECFCs in term of stress history, senescence status, and proliferation capacity may thus account for the differential effect of RSV. Indeed, in contrast to PT-ECFCs, control ECFCs are highly proliferating cells and naive for aging and stress stimuli.

In conclusion, our work identifies premature senescence as a major defect governing angiogenesis in PT-ECFCs. The effect of SIRT1 deficiency underlines the importance of this protein as a key regulator in ECFC homeostasis in early infancy. Our findings lay new grounds for understanding and controlling the increased risk of cardiovascular diseases of individuals born prematurely.

\section{Acknowledgments}

The authors thank the staff of the obstetric departments (Conception and Saint Joseph Hospitals, Marseille).

This work was supported by the French Ministry of Health (PHRC 2725/5789), the Fondation pour la Recherche Médicale (FRM, grant R08046AA), and INSERM. P.F.V. was the recipient of a CAPES postdoctoral scholarship from Brazil.

\section{Authorship}

Contribution: P.F.V. and S.S. designed, conducted the experiments, and interpreted the data; I.L. contributed to sample collection and analysis of clinical data; A.-L.C. contributed to experiments; R.B. contributed to transfection; S.R. contributed to cytometry analysis; J.M. contributed to epigenetic experiments.; S.F. and B.G. contributed to animal experiments; E.T. contributed to clone extraction; M.M. and A.P. provided cord blood samples; U.S. handled funding and supervision and made critical revision of the manuscript; F.A. and F.M. helped with conception, design, and interpretation, analyzed the results, and wrote the paper; F.D.-G. handled funding and supervision of the project, helped with the interpretation of the experiments, and wrote the manuscript; and F.S. coordinated the project and wrote the manuscript.

Conflict-of-interest disclosure: The authors declare no competing financial interests.

The current affiliation for P.F.V. is Health Science Center, Intensive Care Unit, University Hospital, Federal University of Espírito Santo, Vitória, Brazil.

Correspondence: Françoise Dignat-George, Faculté de Pharmacie, INSERM UMR_S 1076, 27 Bd Jean Moulin 13385, Marseille Cedex 05 France; e-mail: francoise.dignat-george@univ-amu.fr.

\section{References}

1. Barker DJ, Winter PD, Osmond C, Margetts B, Simmonds SJ. Weight in infancy and death from ischaemic heart disease. Lancet. 1989;2(8663): 577-580.

2. Ligi I, Grandvuillemin I, Andres V, Dignat-George $\mathrm{F}$, Simeoni U. Low birth weight infants and the developmental programming of hypertension: a focus on vascular factors. Semin Perinatol. 2010;34(3):188-192.

3. Lazdam M, de la Horra A, Pitcher A, et al. Elevated blood pressure in offspring born premature to hypertensive pregnancy: is endothelial dysfunction the underlying vascular mechanism? Hypertension. 2010;56(1):159-165

4. Asahara T, Kawamoto A. Endothelial progenitor cells for postnatal vasculogenesis. Am J Physiol Cell Physiol. 2004;287(3):C572-C579.

5. Kawamoto A, Asahara T. Role of progenitor endothelial cells in cardiovascular disease and upcoming therapies. Catheter Cardiovasc Interv. 2007;70(4):477-484.

6. Hill JM, Zalos G, Halcox JP, et al. Circulating endothelial progenitor cells, vascular function, and cardiovascular risk. $N$ Engl J Med. 2003;348(7): 593-600.

7. Schmidt-Lucke C, Rössig L, Fichtlscherer S, et al. Reduced number of circulating endothelial progenitor cells predicts future cardiovascular events: proof of concept for the clinical importance of endogenous vascular repair. Circulation. 2005; 111(22):2981-2987.
8. Werner N, Nickenig G. Endothelial progenitor cells in health and atherosclerotic disease. Ann Med. 2007;39(2):82-90.

9. Hwang HS, Maeng YS, Park YW, Koos BJ, Kwon YG, Kim YH. Increased senescence and reduced functional ability of fetal endothelial progenitor cells in pregnancies complicated by preeclampsia without intrauterine growth restriction. $A m \mathrm{~J}$ Obstet Gynecol. 2008;199(3):259.e1-259.e7.

10. Ingram DA, Lien IZ, Mead LE, et al. In vitro hyperglycemia or a diabetic intrauterine environment reduces neonatal endothelial colonyforming cell numbers and function. Diabetes. 2008;57(3):724-731.

11. Kwon J-Y, Maeng Y-S, Kwon Y-G, Kim YH, Kang $\mathrm{MH}$, Park YW. Decreased endothelial progenitor cells in umbilical cord blood in severe preeclampsia. Gynecol Obstet Invest. 2007;64(2):103-108.

12. Borghesi A, Massa M, Campanelli R, et al Circulating endothelial progenitor cells in preterm infants with bronchopulmonary dysplasia. $A m \mathrm{~J}$ Respir Crit Care Med. 2009;180(6):540-546.

13. North BJ, Sinclair DA. The intersection between aging and cardiovascular disease. Circ Res. 2012;110(8):1097-1108.

14. Minamino $T$, Miyauchi $H$, Yoshida $T$, Ishida $Y$, Yoshida H, Komuro I. Endothelial cell senescence in human atherosclerosis: role of telomere in endothelial dysfunction. Circulation. 2002; 105(13):1541-1544
15. Chen J, Brodsky SV, Goligorsky DM, et al. Glycated collagen I induces premature senescence-like phenotypic changes in endothelial cells. Circ Res. 2002;90(12): 1290-1298.

16. Imanishi T, Hano T, Sawamura T, Nishio I. Oxidized low-density lipoprotein induces endothelial progenitor cell senescence, leading to cellular dysfunction. Clin Exp Pharmacol Physiol. 2004;31(7):407-413.

17. Ligi I, Simoncini S, Tellier E, et al. A switch toward angiostatic gene expression impairs the angiogenic properties of endothelial progenitor cells in low birth weight preterm infants. Blood. 2011;118(6):1699-1709.

18. Korff T, Kimmina S, Martiny-Baron G, Augustin HG. Blood vessel maturation in a 3-dimensional spheroidal coculture model: direct contact with smooth muscle cells regulates endothelial cell quiescence and abrogates VEGF responsiveness. FASEB J. 2001;15(2):447-457.

19. Ozturk M, Arslan-Ergul A, Bagislar S, Senturk S, Yuzugullu $\mathrm{H}$. Senescence and immortality in hepatocellular carcinoma. Cancer Lett. 2009; 286(1):103-113.

20. Goligorsky MS, Chen J, Patschan S. Stress induced premature senescence of endothelial cells: a perilous state between recovery and point of no return. Curr Opin Hematol. 2009;16(3): 215-219. 
21. Ben-Porath I, Weinberg RA. The signals and pathways activating cellular senescence. Int $J$ Biochem Cell Biol. 2005;37(5):961-976.

22. Stein GH, Drullinger LF, Soulard A, Dulić V. Differential roles for cyclin-dependent kinase inhibitors p21 and p16 in the mechanisms of senescence and differentiation in human fibroblasts. Mol Cell Biol. 1999;19(3):2109-2117.

23. Lim S, Metzger E, Schüle R, Kirfel J, Buettner R. Epigenetic regulation of cancer growth by histone demethylases. Int J Cancer. 2010;127(9): 1991-1998.

24. Bitterman KJ, Anderson RM, Cohen HY, LatorreEsteves M, Sinclair DA. Inhibition of silencing and accelerated aging by nicotinamide, a putative negative regulator of yeast sir2 and human SIRT1. J Biol Chem. 2002;277(47):45099-45107.

25. Avalos JL, Bever KM, Wolberger C. Mechanism of sirtuin inhibition by nicotinamide: altering the $\mathrm{NAD}(+)$ cosubstrate specificity of a Sir2 enzyme. Mol Cell. 2005;17(6):855-868.

26. Minamino T, Komuro I. Vascular cell senescence: contribution to atherosclerosis. Circ Res. 2007; 100(1):15-26.

27. Takahashi T, Kalka C, Masuda $\mathrm{H}$, et al. Ischemiaand cytokine-induced mobilization of bone marrow-derived endothelial progenitor cells for neovascularization. Nat Med. 1999;5(4):434-438.

28. Coleman PR, Hahn CN, Grimshaw M, et al. Stress-induced premature senescence mediated by a novel gene, SENEX, results in an antiinflammatory phenotype in endothelial cells. Blood. 2010;116(19):4016-4024.

29. Heiss C, Keymel S, Niesler U, Ziemann J, Kelm $\mathrm{M}$, Kalka C. Impaired progenitor cell activity in age-related endothelial dysfunction. J Am Coll Cardiol. 2005;45(9):1441-1448.

30. Donmez G, Guarente L. Aging and disease: connections to sirtuins. Aging Cell. 2010;9(2): 285-290.

31. Blander G, Guarente L. The Sir2 family of protein deacetylases. Annu Rev Biochem. 2004;73: 417-435.

32. Cheng H-L, Mostoslavsky R, Saito S, et al. Developmental defects and p53 hyperacetylation in Sir2 homolog (SIRT1)-deficient mice. Proc Nat Acad Sci USA. 2003;100(19):10794-10799.
33. Mostoslavsky R, Chua KF, Lombard DB, et al. Genomic instability and aging-like phenotype in the absence of mammalian SIRT6. Cell. 2006; 124(2):315-329.

34. Li Y, Tollefsbol TO. p16(INK4a) suppression by glucose restriction contributes to human cellular lifespan extension through SIRT1-mediated epigenetic and genetic mechanisms. PLOS ONE. 2011;6(2):e17421.

35. Kwon H-S, Ott M. The ups and downs of SIRT1. Trends Biochem Sci. 2008;33(11):517-525.

36. Potente M, Ghaeni L, Baldessari D, et al. SIRT1 controls endothelial angiogenic functions during vascular growth. Genes Dev. 2007;21(20): 2644-2658.

37. Chen Z, Shentu TP, Wen L, Johnson DA, Shyy JY. Regulation of SIRT1 by oxidative stressresponsive miRNAs and a systematic approach to identify its role in the endothelium. Antioxid Redox Signal. 2013;19(13):1522-1538.

38. Salminen A, Kaarniranta K, Kauppinen A. Crosstalk between oxidative stress and SIRT1: impact on the aging process. Int J Mol Sci. 2013; 14(2):3834-3859.

39. Jankov RP, Negus A, Tanswell AK. Antioxidants as therapy in the newborn: some words of caution. Pediatr Res. 2001;50(6):681-687.

40. Paschalaki KE, Starke RD, $\mathrm{Hu}$ Y, et al Dysfunction of endothelial progenitor cells from smokers and COPD patients due to increased DNA damage and senescence [published online ahead of print July 29, 2013]. Stem Cells.

41. Potente M, Dimmeler S. Emerging roles of SIRT1 in vascular endothelial homeostasis. Cell Cycle. 2008;7(14):2117-2122.

42. Mattagajasingh I, Kim C-S, Naqvi A, et al. SIRT1 promotes endothelium-dependent vascular relaxation by activating endothelial nitric oxide synthase. Proc Natl Acad Sci USA. 2007;104(37): 14855-14860

43. Takemura A, lijima K, Ota H, et al. Sirtuin 1 retards hyperphosphatemia-induced calcification of vascular smooth muscle cells. Arterioscler Thromb Vasc Biol. 2011;31(9):2054-2062.

44. Tauzin L, Rossi P, Giusano B, et al. Characteristics of arterial stiffness in very low birth weight premature infants. Pediatr Res. 2006; 60(5):592-596.
45. Wu Z, Liu M-C, Liang M, Fu J. Sirt1 protects against thrombomodulin down-regulation and lung coagulation after particulate matter exposure. Blood. 2012;119(10):2422-2429.

46. de Kreutzenberg SV, Ceolotto G, Papparella I, et al. Downregulation of the longevity-associated protein sirtuin 1 in insulin resistance and metabolic syndrome: potential biochemical mechanisms. Diabetes. 2010;59(4):1006-1015.

47. Guarente L. Franklin H. Epstein Lecture: sirtuins, aging, and medicine. N Engl J Med. 2011;364(23): 2235-2244.

48. Lakatta EG, Levy D. Arterial and cardiac aging: major shareholders in cardiovascular disease enterprises: Part I: aging arteries: a "set up" for vascular disease. Circulation. 2003;107(1): $139-146$.

49. Vaitkevicius PV, Fleg JL, Engel JH, et al. Effects of age and aerobic capacity on arterial stiffness in healthy adults. Circulation. 1993;88(4 Pt 1): 1456-1462.

50. Schatteman GC, Dunnwald M, Jiao C. Biology of bone marrow-derived endothelial cell precursors. Am J Physiol Heart Circ Physiol. 2007;292(1): $\mathrm{H} 1-\mathrm{H} 18$.

51. Ben-Shoshan J, George J. Endothelial progenitor cells as therapeutic vectors in cardiovascular disorders: from experimental models to human trials. Pharmacol Ther. 2007;115(1):25-36.

52. Milne JC, Lambert PD, Schenk S, et al. Small molecule activators of SIRT1 as therapeutics for the treatment of type 2 diabetes. Nature. 2007; 450(7170):712-716.

53. Camins A, Sureda FX, Junyent F, et al. Sirtuin activators: designing molecules to extend life span. Biochim Biophys Acta. 2010;1799(10-12): 740-749.

54. Hsieh T-C, Lu X, Guo J, Wu JM. Differential regulation of proliferation, cell cycle control and gene expression in cultured human aortic and pulmonary artery endothelial cells by resveratrol. Int $J$ Mol Med. 2010;26(5):743-749.

55. Wang X-B, Zhu L, Huang J, et al. Resveratrolinduced augmentation of telomerase activity delays senescence of endothelial progenitor cells. Chin Med J (Engl). 2011;124(24):4310-4315. 